\title{
Interrelationship between Liver Hemodynamics and Tumor Metabolism in Liver Metastases: Diagnostic Value of Doppler Perfusion Index Revisited
}

\author{
Alicioglu B. ${ }^{1}$, Sarikaya A. ${ }^{2}$, Bulakbasi N. ${ }^{3}$ \\ ${ }^{1}$ Department of Radiology, Trakya University Medical Faculty, Edirne, Turkey, \\ Department of Radiology, Near East University Medical Faculty, Lefkosa, North \\ Cyprus-Turkey; \\ 2Department of Nuclear Medicine, Trakya University Medical Faculty, \\ Edirne, Turkey; \\ ${ }^{3}$ Department of Radiology, Near East University Medical Faculty, Lefkosa, North \\ Cyprus-Turkey \\ Received December 5, 2012; Accepted April 8, 2013.
}

Key words: Liver neoplasms - Neoplasm metastasis - Positron emission tomography - Ultrasonography - Doppler

\begin{abstract}
It should be expected that the hepatic blood flow increase in the cases with liver metastasis. We aimed to find out if there is a correlation between Doppler parameters and hepatic metabolic activity in oncology patients. 35 patients with hepatic metastases who were identified by $18 \mathrm{~F}$-fluorodeoxyglucose positron emission tomography scan and assessed with Doppler ultrasound were included in this prospective study. Patients with hepatic disease, cardiac dysfunction, dehydration, history of alcoholism, intake of antihypertensive or vasoactive medication were excluded. Volume flow of the proper hepatic artery and the portal vein were measured in the hepatoduodenal ligament by Doppler sonography. Doppler perfusion index (the ratio of the hepatic artery flow to the total liver blood flow) and flow volumes of 31 age matched subjects were compared. Both flow of the proper hepatic artery and portal vein were found to be significantly higher in patients with liver metastasis. The mean Doppler perfusion index value was $0.2 \pm 0.13$ in hepatic metastases whereas $0.13 \pm 0.05$ in control group. Doppler perfusion index was significantly higher in liver metastases $(p=0.008)$. A positive correlation was found between the maximum standardized uptake
\end{abstract}

Mailing Address: Banu Alicioglu, MD., Department of Radiology, Near East University Medical Faculty, Lefkosa, Mersin 10, North Cyprus-Turkey; Mobile Phone: 53383706 76; Fax: 9039222364 61; e-mail: alicioglu.b@gmail.com 
value of the liver and flow volume of the proper hepatic artery $(r=0.774, p=0)$. Blood flow of the proper hepatic artery and Doppler perfusion index correlates with hepatic standardized uptake value. Flow measurements of the liver may become an important parameter for selecting patients for further positron emission tomography scan and following-up the response after systemic and local therapeutic procedures.

\section{Introduction}

Liver metastasis is a common consequence of many carcinomas. The detection of hepatic metastases is necessary for exact staging and planning of the treatment. The recent technological improvements have enabled quantitative imaging of hepatic parenchymal and tumoral blood flow. The demonstration of the altered hepatic hemodynamics during the tumoral angiogenesis within the liver metastases may subsequently indicate the presence of metastases even in the absence of manifest tumours. The functional changes are generally detectable before any structural abnormality is apparent, as it is the degree of physiological change rather than its size that determines whether an abnormality is detected (Fuentes et al., 2002). Quantitative perfusion measures may be used for the characterization of hepatic tumours and their biologic aggressiveness and also monitoring the therapeutic response. Increase in tumoral vascularity and glucose metabolism are prominently important factors in the growth and also detection of many tumours (Miles and Williams, 2008; Haider et al., 2010). For correct staging and treatment optimization, the resolving capacity of imaging modalities needs to be improved. However the use of these imaging tools are not common because of their high cost, lower availability and, the necessity of ionising radiation or nephrotoxic contrast agent requirements.

Ultrasonography is the most common and first order modality for hepatic imaging because of its non-invasive and low cost feature. However, sonography is operator dependent and has limited sensitivity in the detection of small tumour nodules; moreover parts of the liver remain non-visible in certain patients (Bipat et al., 2005). Although colour and power Doppler US has increased its sensitivity in the detection of the liver lesions, they did not reach the level of sensitivity of contrast enhanced cross sectional imaging modalities (CT or MRI).

To assess the altered hepatic hemodynamics due to tumoral angiogenesis of liver metastasis, hepatic perfusion index ( $\mathrm{HPI})$ which is the ratio of the hepatic artery flow to the total liver blood (hepatic artery + portal vein) flow was first investigated by dynamic scintigraphy and found to be abnormal in $94 \%$ of colorectal cancer patients with liver metastases (Totman et al., 2005). Later Doppler parameter index (DPI) measured by Doppler US was introduced and it was postulated that even early small hepatic metastases may induce alterations of hepatic blood flow (Cooke et al., 1987; Leen et al., 1991, 1994, 
1997a; Fong, 2000). The advantage of DPI is, that it is an inexpensive and non invasive method, and it does not require usage of contrast material or ionizing radiation to investigate hemodynamic alterations of the liver (Fong, 2000). In recent years, there is an increase in combined multiparametric imaging approach to study the relationship between vascularisation and metabolism. FDG-PET/CT (fluorodeoxyglucose-positron emission tomography - computed tomography) is capable to demonstrate abnormal metabolic activity at the molecular level in organs that as yet do not show an abnormal appearance based on morphologic criteria (Kapoor et al., 2004).

The aim of this study is to investigate hepatic perfusion changes of the patients with liver metastasis by means of DPI measurements and find out any correlation between liver hemodynamics and tumour metabolism measured by FDG-PET/CT scan and to research any complementary diagnostic or predictive role in the clinical management.

\section{Material and Methods}

The study was approved by the Local Ethics Committee; informed written consent was obtained from all the patients. Thirty-five patients ( 21 male and 14 female) with hepatic metastases were included in this prospective study. Patients were selected from the FDG-PET/CT unit of our University Hospital where they were scanned for staging purpose because of their proven malignancies. Primary origins were; lung cancer in 10 patients, colorectal cancer in nine patients, breast cancer in five patients, ovarian malignancy in three patients, thyroid medullary carcinoma in one patient, gastric carcinoma in one patient, anal carcinoma in one patient, pancreas carcinoma in one patient and unknown process in two patient. The mean patient's age was $59.54 \pm 11.16$ (ranging 31 to79). The patients did not have clinical evidence of liver disease, cardiac dysfunction and dehydration. The patients did not have a history of alcoholism or intake of antihypertensive or vasoactive medication.

Control group consisted of 31 patients (15 male and 16 female); the absence of liver metastasis was proven by PET/CT. There was no evidence of any hepatic disease, cardiac dysfunction, dehydration. They did not have a history of alcoholism or intake of antihypertensive or vasoactive medication. The mean age of the control group was $55.77 \pm 12.32$ (ranging 32 to 63 ).

FDG-PET/CT was performed using a hybrid system (General Electric, Discovery LS, Milwaukee, WI, USA) after i.v. injection of $5.4 \mathrm{MBq} / \mathrm{kg}$ of FDG with a minimum $6 \mathrm{~h}$ fasting blood glucose level of less than $140 \mathrm{mg} / \mathrm{dl}$. Data acquisitions were performed within 60-120 min after injection. Oral contrast was only given to the patients for the CT portion of the study. Quantification of the tumour metabolic activity was obtained using the maximum standardized uptake value (SUVmax) normalized to body weight. Pathologic 18F-FDG accumulation was identified by focal tracer uptake that exceeded that of the normal hepatic parenchyma (Figure 1). Tumour lesions were identified as areas of pathologically increased 18F-FDG 


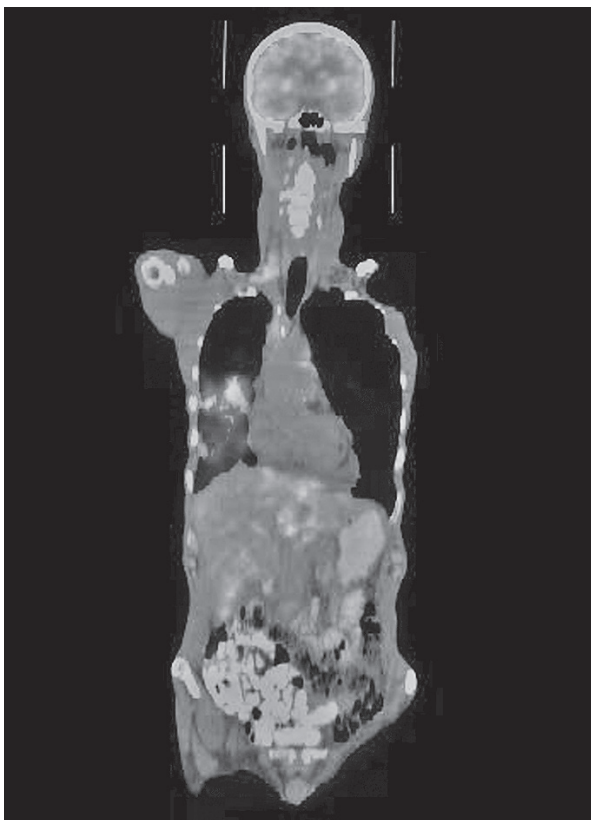

Figure 1 - 56-years-old male patient with lung cancer. Multiple hepatic metastases are seen on PET/CT scan. The metastases were not obvious on gray-scale US. uptake. Regions of interest (ROls) were drawn on the area of abnormal 18F-FDG uptake corresponding to the tumour.

Patients who were detected to have metastasis in the liver were examined by CDUS (Clinical Data Update System). Interval between FDG-PET/CT and CDUS was 1-2 days. CDUS examinations were performed by the same experienced radiologist. US system (Esaote S.p.A., Italy) was used for CDUS examination. Following 8 hours of starvation the patients were examined on supine or left decubitus position holding their breath. First the liver parenchyma was scanned on gray-scale and the presence and the segmental location of metastatic lesions were noted. Secondly, both proper hepatic artery (PHA) and portal vein (PV) were identified longitudinally along their course in the hepatoduodenal ligament. Spectral

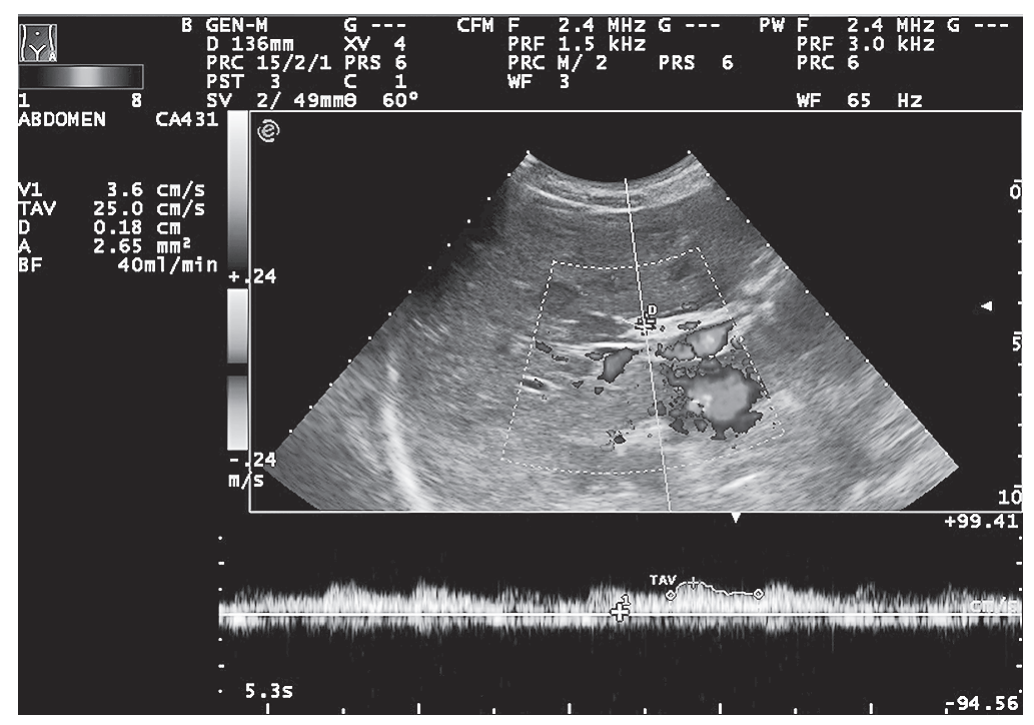

Figure 2 - CDUS of the same patient. Despite the presence of large number of metastases the hepatic artery blood flow is not increased. 


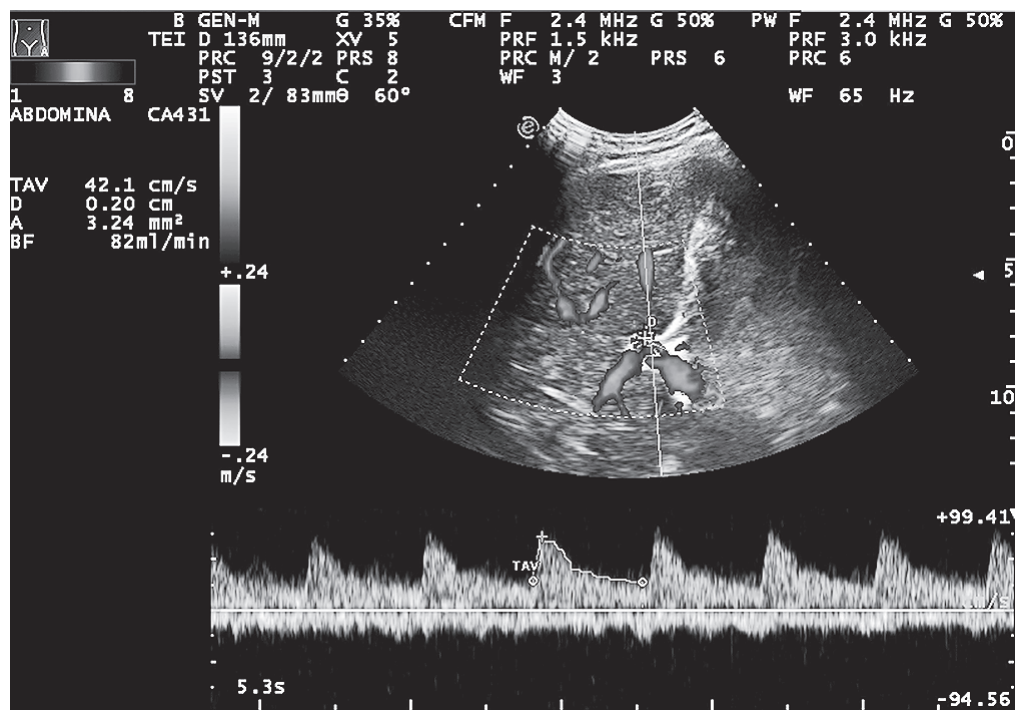

Figure 3 - Increase of the hepatic arterial blood flow in a Hodgkin lymphoma patient having multiple liver metastases.

analysis was determined based on the technique described in the previous studies (Cooke et al., 1987; Leen et al., 1991, 1994, 1997a; Fong, 2000; Oktar et al., 2006) (Figures 2 and 3). Volume flows of the proper hepatic artery (PHAF) and portal vein (PVF) were calculated from the product of the time-averaged blood velocity and the diameter for each vessel. The measurements were recorded electronically. DPI value was calculated by the formula: $\mathrm{DPI}=\mathrm{PHAF} /(\mathrm{PHAF}+\mathrm{PVF})$.

FDG-PET/CT scans of the patients were analyzed retrospectively. SUVmax values of the metastases and normal liver parenchyma were measured. To measure SUVmax, a $2 \mathrm{D}$ region of interest $(\mathrm{ROI})$ is positioned centrally within the metastatic tumour on the Workstation. Another same size ROI was placed on a normal liver parenchyma reflecting SUVmax of the liver parenchyma. Maximum SUVmax values were considered for statistical analyze. Finally the presence of additional metastases (lymph node, lung, brain, bone) was recorded. Statistical analysis was performed using a commercially available statistical software program. For comparisons of two groups, independent two-sample $t$-test was performed. Bivariate correlation was used to assess the statistical correlations between DPI value and liver SUVmax and SUVmax of the metastases. For all statistical tests, the level of significance was accepted at $p<0.05$.

\section{Results}

Descriptive analysis of the study group and controls is given in Table 1.

In patients with liver metastase(s) mean DPI value was $0.2 \pm 0.13$; in control group mean DPI value was $0.13 \pm 0.05$; and DPI was significantly higher in liver metastases group $(p=0.008)$. 
Table 1 - Descriptive statistics of the patients and controls

\begin{tabular}{|c|c|c|c|c|c|}
\hline \multirow{2}{*}{ Parameter } & PHAF & PVF & DPI & $\begin{array}{l}\text { SUVmax of } \\
\text { the liver }\end{array}$ & $\begin{array}{l}\text { SUVmax of } \\
\text { the metastatic } \\
\text { lesion }\end{array}$ \\
\hline & $\begin{array}{c}(\mathrm{ml} / \mathrm{min}) \\
\operatorname{mean} \pm \mathrm{SD} \\
(\min -\max )\end{array}$ & $\begin{array}{c}(\mathrm{ml} / \mathrm{min}) \\
\operatorname{mean} \pm \mathrm{SD} \\
(\min -\max )\end{array}$ & $\begin{array}{r}\operatorname{mean} \pm \text { SD } \\
(\min -\max )\end{array}$ & $\begin{array}{r}\operatorname{mean} \pm \text { SD } \\
(\min -\max )\end{array}$ & $\begin{array}{r}\operatorname{mean} \pm S D \\
(\min -\max )\end{array}$ \\
\hline Patients $(n=35)$ & $\begin{array}{c}163.9 \pm 147.7 \\
(25-596)\end{array}$ & $\begin{array}{c}822.1 \pm 643.3 \\
(112-2745)\end{array}$ & $\begin{array}{l}0.2 \pm 0.13 \\
(0.02-0.5)\end{array}$ & $\begin{array}{c}2.9 \pm 1.17 \\
(1.8-6.8)\end{array}$ & $\begin{array}{l}9.0 \pm 5.16 \\
(2.9-25.7)\end{array}$ \\
\hline Controls $(n=31)$ & $\begin{array}{c}61.9 \pm 29.5 \\
(20-155)\end{array}$ & $\begin{array}{c}511.03 \pm 365.04 \\
(93-1591)\end{array}$ & $\begin{array}{l}0.13 \pm 0.05 \\
(0.03-0.18)\end{array}$ & - & - \\
\hline
\end{tabular}

PHAF - flow volume of the proper hepatic artery; PVF - flow volume of the portal vein; DPI - Doppler perfusion index; SUV - standardized uptake value

DPI value was significantly correlated with the liver SUVmax $(r=0.461, p=0.003)$. Figure 4 displays the relationship between DPI and liver SUVmax. Accordingly $21 \%$ variance of the liver SUVmax was found to be accounted by DPI. But there was not any significant correlation between DPI value and SUVmax of the metastases $(p=0.101)$.

Among the patient group, 10 cases of liver metastases were not visible on grayscale US, whereas in 25 patients the metastases were visible on gray-scale US. DPI values significantly differed between the cases with sonographically detectable and undetectable metastases $(p=0.015)$. Mean DPI value was $0.23 \pm 0.13$ in overt

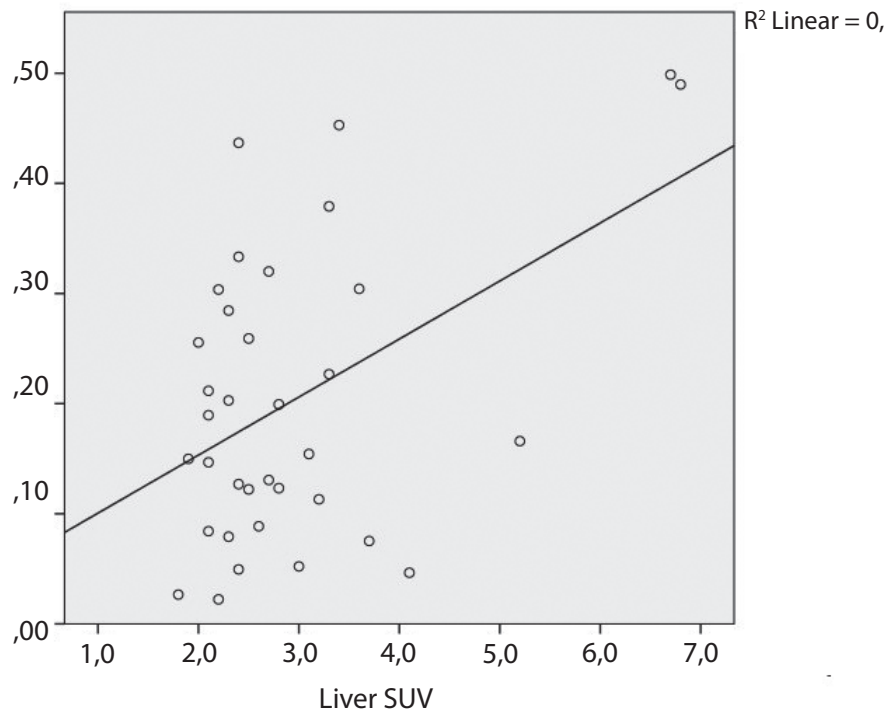

Figure 4 - Scatter plot showing the relation between DPI value and liver SUV of the patients with liver metastases.

Alicioglu B.; Sarikaya A.; Bulakbasi N. 
metastases; whereas $0.12 \pm 0.1$ in occult metastases $(p=0.02)$. However, DPI value of the occult metastases and controls was not significantly different $(p>0.05)$.

DPI value was not different between patients with and without extra-hepatic metastasis $(p>0.05)$.

\section{Discussion}

Doppler perfusion index have been found to be increased in the liver metastases of colorectal tumours and could be used as an indicator of metastases which are sonographically small or undetectable (Leen et al., 1991, 1994, 1997a; Fong, 2000; Kopljar et al., 2004; Oktar et al., 2006). Beyond diagnostic capability, abnormal DPI values were also reported to predict clinical outcome, lymph node and liver metastases (Cooke et al., 1987; Leen et al., 1997b, 2000; Warren et al., 1998; Oppo et al., 2000; Fuentes et al., 2002; Miles et al., 2011). The method used in this study to measure hepatic arterial blood flow differs from that originally described. We studied proper hepatic artery which directly feeds liver and gives only cystic artery; in that location the possible variations are replaced left hepatic artery from left gastric artery or replaced right hepatic artery from superior mesenteric artery whose incidences are about $20 \%$. In case of variant patterns, the lobes may receive blood supply from the superior mesenteric artery, left gastric artery, aorta, or other visceral branches (Hiatt et al., 1994). Our DPI values were still comparable with the previous results (Leen et al., 1991, 1994, 1997a; Fong, 2000; Guadagni et al., 2000; Oktar et al., 2006). Due to the small size of our study group, it was not possible to determine a distinct cut-off value but DPI $>0.2$ could be assessed as strong possibility of metastasis; DPI value between 0.13 and 0.2 indicates probable possibility of metastasis and DPI $<0.13$ indicates strong possibility of absence of metastasis.

Doppler perfusion index value was mainly studied in colorectal cancer metastases in the previous literature. They all concluded that increase in the hepatic artery blood flow and decrease in the portal vein blood flow results by an increased DPI value (Leen et al., 1991, 1994, 1997a; Fong, 2000; Oktar et al., 2006; Kyriakopoulou et al., 2008). Accordingly DPI value of the liver with metastases was found >0.3-0.5; benign diseases had DPI value $<0.15$ (Leen et al., 1991; Oppo et al., 2000; Kyriakopoulou et al., 2008). Our study group had heterogeneous malignancies but all the tumours had to provide their own blood supply to promote growth; this process is called angiogenesis or neovascularisation and is supported by numerous polypeptide growth factors and factors promoting tumour angiogenesis (vascular endothelial growth factor and basic fibroblast growth factor) (Kapoor et al., 2004). DPI value was found to be correlated with the liver SUV as we expected. Within the liver the new blood vessels are derived mostly from the arterial system (Fuentes et al., 2002). It is reasonable to hypothesise that the metabolic requirements of tumours go parallel with changes in tumour hemodynamics. Similarly CT measurements of arterial perfusion and arterial 
phase enhancement have been found to be correlated with DPI (Fuentes et al., 2002). However the relationship between tumoral blood flow and metabolism is not always parallel. Incongruity of tumour perfusion and metabolism has been noted in previous studies investigating the relationship between perfusion and metabolism of tumours (Miles et al., 2011). The balance between liver blood flow and metabolism would play an important sign of the biological behaviour of the metastases. Rapid proliferation of large tumours outgrows their vascular supply, resulting in ischemia and necrosis of the tumour (Kapoor et al., 2004). The arterial and venous supplies to the liver are not independent systems; there can be several communications between vessels, including transsinusoidal, vassal, tumoral and plexal-peribiliary routes (Haider et al., 2010). But generally portal connections are the draining veins (Fuentes et al., 2002). Finally, the increased arterialisation of the metastases brings about increased DPI. Thus, this phenomenon could explain positive correlation between DPI and the liver SUV. Although our results indicate a moderate correlation between liver SUV and DPI, only $21 \%$ variance of the liver SUV was found to be accounted by DPI, leaving the rest of the variance unexplained. Doppler US assesses only total blood flow of the liver; namely increase in the size of the liver or cardiac output or body weight of the patient which result in alteration of the liver blood flow. On the other hand FDG-PET/CT displays metabolic activity of the liver at molecular level and it does not depend on the cardiovascular status or size of the liver. Therefore the increased DPI value has to be carefully assessed in the presence of hepatomegaly.

FDG-PET/CT scan allows imaging of glucose metabolism; it can lead to early detection of the tumour by detecting abnormal tumour metabolism prior to the appearance anatomic changes and localization of tumour (Kinkel et al., 2002; Bipat et al., 2005). The rate of uptake of FDG by the tumour cells is proportional to their metabolic activity (Fuentes et al., 2002). The SUV is a semi-quantitative assessment of the radiotracer uptake from a static (single point in time) PET image. Glucose uptake of the tumour cells will be followed by micro-vascular processes. The lack of correlation between DPI and SUVmax is therefore reasonable. FDG-PET/CT reflects molecular tracer concentration in the tissues, whereas total blood flow of the liver is assessed by Doppler US. Thus one should not expect that total blood flow of the liver reflects metabolic activity of a tumour within the liver.

In contrast to the literature, we were not able to demonstrate that DPI could discriminate occult metastases; DPI was not found to be different in patients with and without extra-hepatic metastases. Heterogeneity of the malignancies and the different scanning methods used for the comparison could explain discrepancy. When high-cost, nephrotoxic contrast medium requirement and ionizing radiation; and high possibility of the need of re-scanning of these patients is considered US with DPI value could be a predictor to select the patients who have undergone further imaging methods. Higher DPI can predict the liver SUV, 
and predict diffuse liver metastases which are indistinguishable on conventional scanning techniques.

The repeatability and reproducibility of the Doppler US measurements is the major handicap of US imaging which is highly operator dependent. We could not find any information about intra-observer variance of measurements of HAF and PVF to calculate DPI; only one study in literature reports that there is no intraobserver variance (Oktar et al., 2006). Besides the possibility of anatomical variances; individual hemodynamic variances even the phase of inspiration alters the flow measurements.

As a conclusion, multimodality imaging studies of perfusion and metabolism of the tumours has an increasing role in oncology patients. Increased arterial flow is one of the major radiological signs for the presence of liver metastases and is related to increased metabolic activity of the liver. Measurements of liver blood flow by Doppler US is easy, repeatable, cheap, radiation or nephrotoxic contrast agent safe and should be incorporated to standard follow-up protocol of oncology patients. DPI could not discriminate occult liver metastases from normal liver. However, DPI could be used to increase diagnostic capacity of US and select patients for further imaging modalities and follow-up the response after systemic and local therapeutic procedures. We propose measurements of the proper hepatic artery to be less influenced from anatomical variations.

\section{References}

Bipat, S., Leeuwen, M. S., Comans, E. F. I., Pijl, M. E., Bossuyt, P. M., Zwinderman, A. H., Stoker, J. (2005)

Colorectal liver metastases: CT, MR imaging, and PET for diagnosis-meta analysis. Radiology 126, 123-131.

Cooke, D. A., Parkin, A., Wiggings, P., Robinson, P. J., Giles, G. R. (1987) Hepatic perfusion index and THA evolution of liver metastases. Nucl. Med. Commun. 8, 970-974.

Fong, Y. (2000) Doppler perfusion index in colorectal cancer. Lancet 355, 5-6.

Fuentes, M. A., Keith, C. J., Griffiths, M., Durbridge, G., Miles, K. A. (2002) Hepatic haemodynamics: Interrelationships between contrast enhancement and perfusion on CT and Doppler perfusion indices. Br. J. Radiol. 75, 17-23.

Guadagni, S., Pizzutilli, A., Mancini, E., Varrone, A., Palumbo, G., Amicucci, G., Perri, S., Deraco, M., Fiorentini, G. (2000) Significance of duplex/color Doppler sonography in hepatic arterial chemotherapy for patients with liver metastases from colorectal carcinoma. Eur. J. Surg. Oncol. 26, 381-386.

Haider, M. A., Farhadi, F. A., Milot, L. (2010) Hepatic perfusion imaging: Concepts and application. Magn. Reson. Imaging Clin. N. Am. 18, 465-475.

Hiatt, J. R., Gabbay, J., Busuttil, R. W. (1994) Surgical anatomy of the hepatic arteries in 1000 cases. Ann. Surg. 220, 50-52.

Kapoor, V., McCook, B. M., Torok, F. S. (2004) An introduction to PET-CT imaging. Radiographics 24, 523-543.

Kinkel, K., Lu, Y., Both, M., Warren, R. S., Thoeni, R. F. (2002) Detection of hepatic metastases from cancers of the gastrointestinal tract by using noninvasive imaging methods (US, CT, MR imaging, PET): A metaanalysis. Radiology 224, 748-756.

Kopljar, M., Brkljacic, B., Doko, M., Horzic, M. (2004) Nature of Doppler perfusion index changes in patients with colorectal cancer liver metastases. J. Ultrasound Med. 23, 1295-1300.

Kyriakopoulou, K., Antoniou, A., Fezoulidis, I. V., Kelekis, N. L., Dalekos, G. N., Vlychou, M. (2008) The role of 
Doppler perfusion index as screening test in the characterisation of focal liver lesions. Dig. Liver Dis. 40, 755-760.

Leen, E., Goldberg, J. A., Robertson, J., Sutherland, G. R., McArdle, C. S. (1991) The use of duplex sonography in the detection of colorectal hepatic metastases. Br. J. Cancer 63, 323-325.

Leen, E., Angerson, W. J., Wotherspoon, H., Moule, B., Cooke, T. G., McArdle, C. S. (1994) Comparison of the Doppler perfusion index and intraoperative ultrasonography in diagnosing colorectal liver metastases. Ann. Surg. 220, 663-667.

Leen, E., Anderson, J. R., Robertson, J., O'Gorman, P., Cooke, T. G., McArdle, C. S. (1997a) Doppler index perfusion in the detection of hepatic metastases secondary to gastric carcinoma. Am. J. Surg. 173, 99-102.

Leen, E., Anderson, W. G., Cooke, T. G., McArdle, C. S. (1997b) Prognostic power of Doppler perfusion index in colorectal cancer. Correlation with survival. Ann. Surg. 223, 199-203.

Leen, E., Goldberg, J. A., Angerson, W. J., McArdle, C. S. (2000) Potential role of Doppler perfusion index in selection of patients with colorectal cancer for adjuvant chemotherapy. Lancet 355, 34-37.

Miles, K. A., Williams, R. E. (2008) Warburg revisited: Imaging tumour blood flow and metabolism. Cancer Imaging 8, 81-86.

Miles, K. A., Williams, R. E., Yu, D., Griffiths, M. R. (2011) Demonstrating intertumoral differences in vascular-metabolic phenotype with dynamic contrast-enhanced CT-PET. Int. J. Mol. Imaging, doi:10.1155/2011/679473.

Oktar, S. O., Yücel, C., Demirogullari, T., Uner, A., Benekli, M., Erbas, G., Ozdemir, H. (2006) Doppler sonographic evaluation of hemodynamic changes in colorectal liver metastases relative to liver size. J. Ultrasound Med. 25, 575-582.

Oppo, K., Leen, E., Angerson, W. J., McArdle, C. S. (2000) The effect of resecting the primary tumour on the Doppler perfusion index in patients with colorectal cancer. Clin. Radiol. 55, 791-793.

Totman, J. J., O'Gorman, R. L., Kane, P. A., Karani, J. B. (2005) Comparison of THA hepatic perfusion index measured with gadolinium-enhanced volumetric MRI in controls and in patients with colorectal cancer. Br. J. Radiol. 78, 105-109.

Warren, H. W., Gallagher, H., Hemingway, D. M., Angerson, W. J., Bessent, R. G., Wotherspoon, H., McArdle, C. S., Cooke, T. G. (1998) Prospective assessment of the hepatic perfusion index in patients with colorectal cancer. Br.J. Surg. 85, 1708-1712. 